\title{
Council of Europe dismisses claim that abortion in Italy is harder because more doctors are objecting
}

We are sorry that two errors occurred in editing this letter by Carlo Petrini (BMJ 2016;354:i4327, doi:10.1136/bmj.i4327) for publication.
In reference 3 the author is Ministero della Salute [not della Salute, as published].

In reference 4 Gazzetta Ufficiale della Repubblica Italiana is the journal [not the author, as published]. 